\title{
Influence of gender on muscle fatigue during dynamic knee contractions
}

\author{
Chiharu FuJISAWA, MSc, PT ${ }^{1,2}$, Akira TAMAKI, PhD, $\mathrm{PT}^{2}$, Eiji YAMADA, PhD, $\mathrm{PT}^{3}$ and \\ Hirofumi MATSUOKA, PhD, MD ${ }^{4}$ \\ ${ }^{1)}$ Shinko Hospital Rehabilitation Center \\ ${ }^{2)}$ Graduate School of Health Science, Hyogo University of Health Sciences \\ ${ }^{3)}$ Kaisei General Hospital, Department of Physical Therapy, Joint Surgical Center \\ ${ }^{4)}$ Shinko Hospital, Department of Respiratory Medicine
}

\begin{abstract}
Purpose: The purpose was to compare quadriceps muscle fatigue and change in surface electromyogram (sEMG) spectral power, muscle thickness, and peak torque (normalized by body weight) in men and women during isokinetic knee contractions. Methods: Nineteen healthy volunteers (10 men, 9 women) participated. The volunteers performed 32 consecutive maximal isokinetic knee contractions for peak torque and muscle fatigue index (FI). The sEMG data were analyzed using wavelet analysis for median frequency (MF). Muscle thickness was measured using ultrasonography. Results: Men had a significantly higher FI, peak torque $(\mathrm{Nm} / \mathrm{kg})$, muscle thickness than women $(\mathrm{p}<0.05)$. A significant linear decreased MF slope in the vastus lateralis was observed $(\mathbf{p}<0.05)$ in men than in women. There was no significant difference in MF slope in the vastus medialis between men and women. Conclusion: During muscle fatigue assessment, men had a significantly greater muscle thickness, knee extension peak torque, and a higher decrease of MF slope than women. Our results indicate that specific muscle fatigue observed during repeated muscle knee contractions is significantly influence by gender and affects MF slope, knee extension peak torque, and muscle thickness.
\end{abstract}

Key words: muscle fatigue, gender difference, quadriceps femoris

(Phys Ther Res 20: 1-8, 2017)

Muscle mass, muscle fiber type, and muscle recruitment pattern $^{1,2)}$ are strong determinants of quadriceps muscle force-generation. Such muscle force generation is significantly influenced by gender-5) ${ }^{3-5}$ general, the knee extensor muscle forces are significantly higher in men than in women. However, men have been shown to exhibit a faster rate of knee extensor muscle fatigue than women ${ }^{6,7)}$. This is likely due to a greater muscle mass and higher percentage of fast-twitch muscle fibers ${ }^{8,9)}$ and, the pattern of muscle recruitment $^{1}$. These factors are associated with muscle fatigue. However, few studies have sought to clarify whether

Received: November 11, 2015

Accepted: October 7, 2016

Advance Publication by J-STAGE: February 24, 2017

Correspondence to: Chiharu Fujisawa, Shinko Hospital Rehabilitation Center, 1-4-47 Wakinohamacho, Chuo-ku, Kobe, Hyogo 651-0072, Japan

\# e-mail: fairbairn.ww2@gmail.com

doi: 10.1298/ptr.E9889 there is an influence of gender on muscle mass, the pattern of muscle recruitment and recruitment of fast-twitch muscle fibers during muscle fatigue assessment.

Surface electromyogram (sEMG) is the most widely used noninvasive method to measure muscle activity, muscle recruitment pattern, and fatigue during isometric muscle contraction. It is well known that traditional spectral estimation techniques cannot be assessed in a non-stationary parameter. These techniques assume that the signals are stationary and therefore they cannot cater for the movement component and noise. However, non-stationary parameters have been assessed (e.g., isokinetic or isotonic muscle contractions and cycle-ergometer) by wavelet analysis ${ }^{11,12)}$. Change in sEMG spectral power during dynamic knee contractions have been reported to decrease median frequency (MF) in healthy volunteers ${ }^{13-15)}$. Fast-twitch muscle fibers exhibit higher conduction velocity and increase to a higher $\mathrm{MF}^{16,17)}$. Interestingly, $\mathrm{MF}$ is significantly associated with muscle fiber type compositions ${ }^{16)}$. Little is known about the 
relationship between muscle fatigue, muscle mass, and the pattern of muscle recruitment during dynamic knee contractions and their association with gender. Moreover, there are few studies that have attempted to elucidate the key factors of muscle fatigue between males and females.

Although there are currently insufficient data to support a causal relationship between muscle fatigue and skeletal muscle mass or change in MF between males and females, it is conceivable that this could be measured through sEMG spectral power and ultrasonography. Assessing the role of gender in muscle fatigue cannot be estimated without further studies of its measurement in muscle thickness and sEMG during isokinetic muscle contractions. We measured the sEMG and ultrasonography findings and isokinetic muscle strength in healthy volunteers to determine the influence of gender on the change in MF, muscle thickness, and muscle fatigue. We hypothesized that gender difference in muscle fatigue is associated with decrease in the MF slope of sEMG spectral power and muscle thickness.

In general, gender difference in muscle fatigue has been considered to be affected by muscle mass, muscle fiber type, and muscle recruitment pattern. However, their factors has not been examined during muscle fatigue assessment at almost the same time. The purpose of this study was to compare quadriceps muscle fatigue and change in sEMG spectral power, muscle thickness of the vastus lateralis (VL), and vastus medialis (VM) and knee extension peak torque during maximal knee contractions between men and women.

\section{Methods}

\section{Design and setting}

The study used a cross-sectional study design, in which healthy volunteers from the Hyogo University of Health Sciences laboratory participated.

\section{Subjects}

Nineteen healthy volunteers were recruited between December 2013 and September 2014. The exclusion criteria were experience with resistance training beyond the previous six months and individuals with a history of cardiovascular disease, diabetes, hypertension, or injury. All volunteers provided written informed consent, and the study was approved by the institutional review board ethics committee of Hyogo University of Health Sciences. This was study, protocol no. 13018. We determine sample size using Gpower 3.1.7, based on a pilot study of five healthy men and five healthy women in the present study. A power calculation determined that 16 healthy volunteers (eight men and eight women) would result in a type 1 error rate of 5\% and a type 2 error rate of $20 \%$ ( $80 \%$ power). To account for data error and participant drop-out, we included 20 healthy volunteers (10 healthy men and 10 healthy women). Base- line characteristics included 19 healthy [ 10 healthy men (age $21.4 \pm 0.49$ years, height $170.6 \pm 5.21 \mathrm{~cm}$, weight $66.11 \pm 9.62 \mathrm{~kg}$ ) and 9 healthy women (age $20.8 \pm 1.33$ years, height $158.4 \pm 3.83 \mathrm{~cm}$, weight $48.57 \pm 4.0 \mathrm{~kg}$ )] volunteers (data from one volunteer were not included because she was unable to complete the study protocol.). All assessment procedures in this study were administered to all volunteers by one investigator.

\section{Muscle fatigue assessment}

We measured the knee extensor peak torque and fatigue of the knee extensor muscles using an isokinetic dynamometer (Biodex System Version 3, Biodex Medical, Shirley, NY, USA). Reciprocal concentric isokinetic knee extension and flexion were assessed at an angular velocity of $180^{\circ} \%$ s. Range of motion was a knee angle of $0^{\circ}$ to $90^{\circ}$. Gravity correction was obtained by measuring the torque exerted on the dynamometer resistance adapter with the knee in a relaxed state at $10^{\circ}$ flexion. The volunteers were seated on the Biodex dynamometer and were strapped to the chair in accordance with the Biodex user's manual. Lindstrom et al. ${ }^{18)}$ and Pincevero et al. ${ }^{10)}$ reported a linear reduction in peak torque over a short period of $30 \mathrm{~s}$, ; however, angular velocity of $60^{\circ} \%$ involving $3 \mathrm{~s}$ for knee extension and flexion (knee angle of $0^{\circ}-90^{\circ}$ ) and the low repetition number (10 repetitions) contributed to the high variance in the muscle fatigue data. Angular velocity of $180^{\circ} / \mathrm{s}$ involving $1 \mathrm{~s}$ for knee extension and flexion and 30 repetitions have been reported to contribute to a lower variance in muscle fatigue data compared with angular velocity of $60^{\circ} \%$ s. Therefore, in our study, reciprocal concentric isokinetic knee extension and flexion were assessed at angular velocity of $180^{\circ}$ s. Owing to the high variance in muscle fatigue assessment of the first and final repetitions of knee extension, data from these two repetitions were excluded ${ }^{13)}$. The volunteers performed 32 consecutive maximal isokinetic contractions of the knee extensor and flexor muscles. Isokinetic knee extensor torque data were recorded as absolute values $(\mathrm{Nm})$ and subsequently normalized to skeletal muscle mass $(\mathrm{Nm} / \mathrm{kg})$. We measured the knee extensor peak torque to determine the muscle fatigue. Muscle fatigue was calculated by fatigue index (FI) to yield a present decrease for each isokinetic knee extensor torque, in a method similar to that performed by Pincivero et al. ${ }^{6}$ :

FI $=100-[$ (last five peak torque/ highest five peak torque) $\times 100]$

\section{sEMG measurements}

sEMG was detected from VL and VM of the dominant thigh throughout the muscle fatigue assessment. Before sEMG electrodes were placed on the target muscle, the skin was shaved and cleaned with 95\% alcohol to reduce impedance to less than $5 \mathrm{k} \Omega$. Bipolar sEMG electrodes (Blue Sensor P; Ambu) were placed on the target muscle according to 


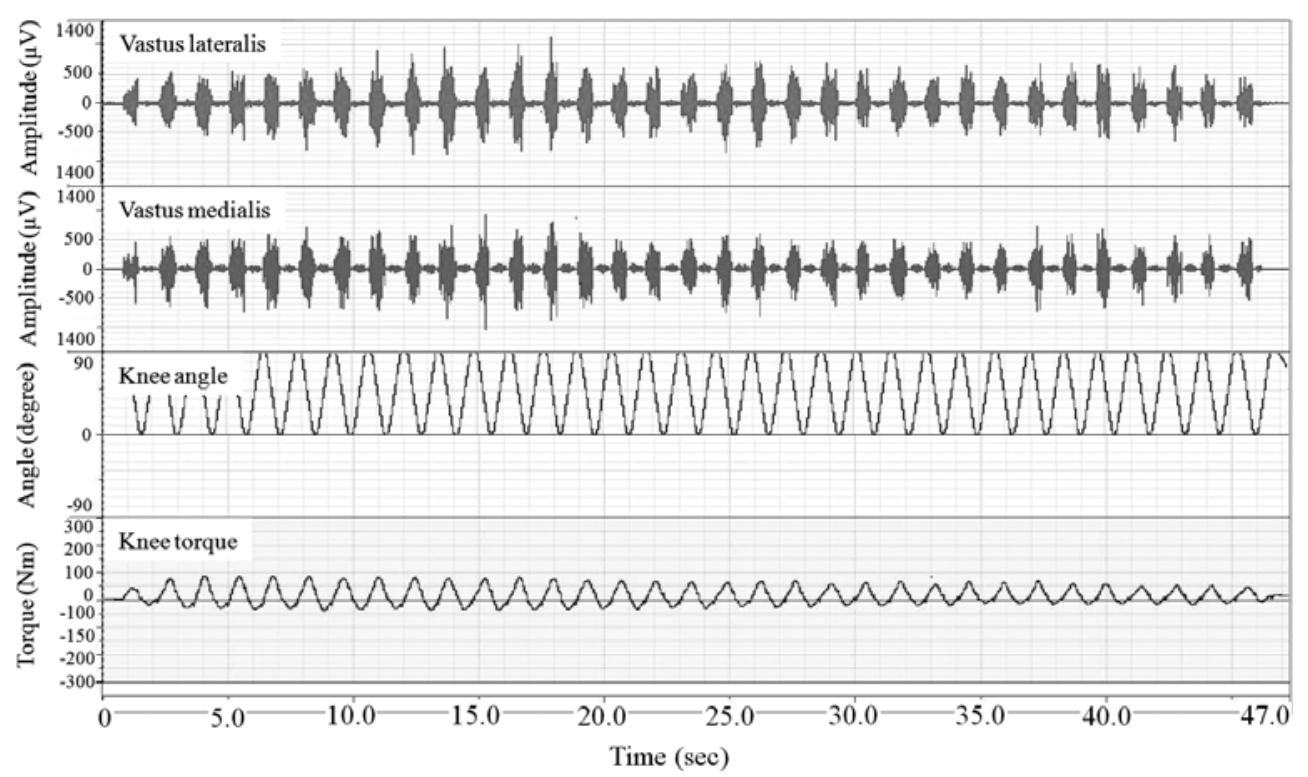

Figure 1. sEMG measurements

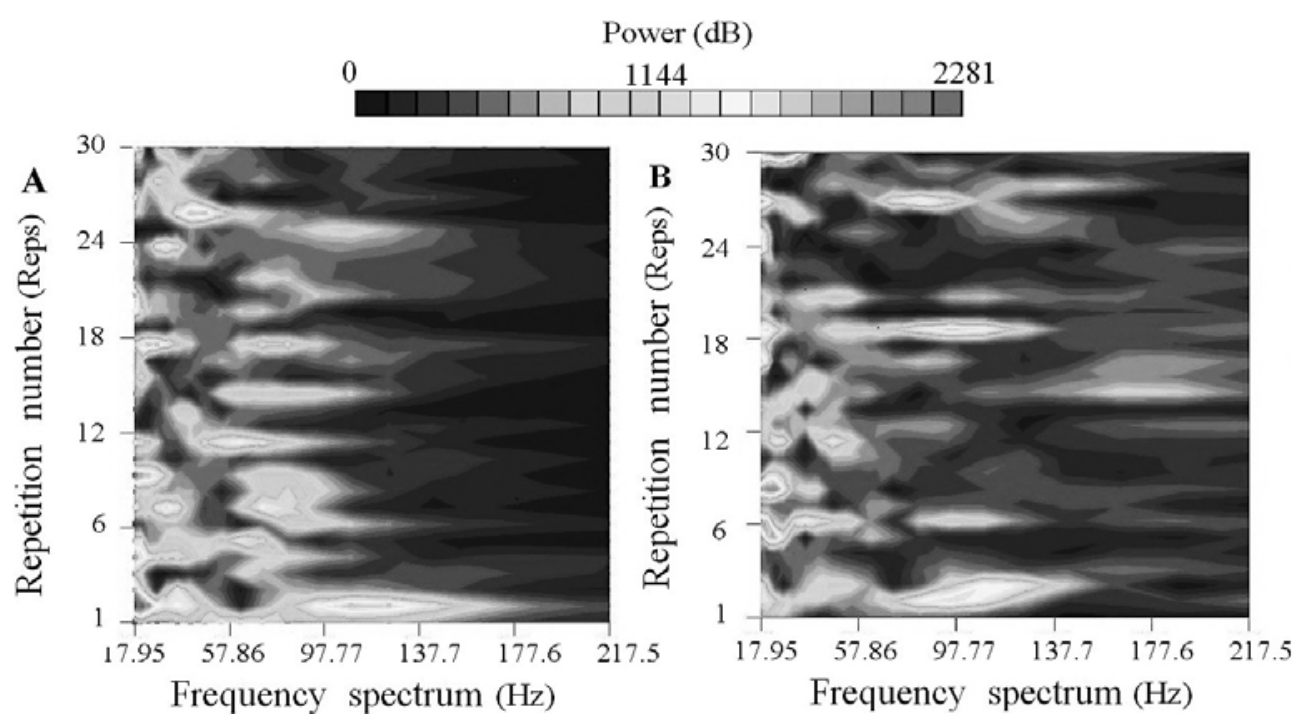

Figure 2. sEMG spectrum

A: Vastus lateralis muscle in sEMG spectrum. B: Vastus mediaris muscle in sEMG spectrum.

the recommendations from the SENIAM project ${ }^{19)}$ i.e., approximately in line with the pennation angle of the muscle fibers. For each muscle, the inter-electrode distance was 30 $\mathrm{mm}$. A ground electrode was placed on the head of the fibula. The sEMG signals were digitized with 16-bit resolution to a digital converter (Myoresearch XP; Noraxon) at a sampling frequency of $1.5 \mathrm{kHz}$ and stored in a personal computer (Tele MyoG2 EM-601; Noraxon) for subsequent analysis (Fig. 1). The sEMG frequency spectrum was between $12.5 \mathrm{~Hz}$ and $200 \mathrm{~Hz}$. For wavelet analysis, KmMercury software (Mediarea support business union) was used. Km-mercury software with Gabor filtering was used to calculate the intensity of the wavelet coefficient for the nineteen domains (Fig. 2). The angular information of the Biodex dynamometer was synchronized with the telemetric transmitter during the muscle fatigue assessment. The acceleration and deceleration phases of movement of isokinetic knee extension were excluded (the middle $45 \pm 15^{\circ}$ of the range of motion were included) from each muscle contractions during the muscle fatigue assessment for wavelet analysis. Owing to the high variance in the sEMG data of the first and final (the $1^{\text {st }}$ and the $32^{\text {nd }}$ ) repetitions of maximum knee extension, data from these two repetitions were excluded $^{13)}$. Each knee extension included wavelet analysis for MF.

\section{Muscle thickness}

The muscle thickness of VL and VM on the muscle assessment leg was measured using real-time B-mode ultrasonography (Logiqbook XP enhanced; GE Healthcare) with 


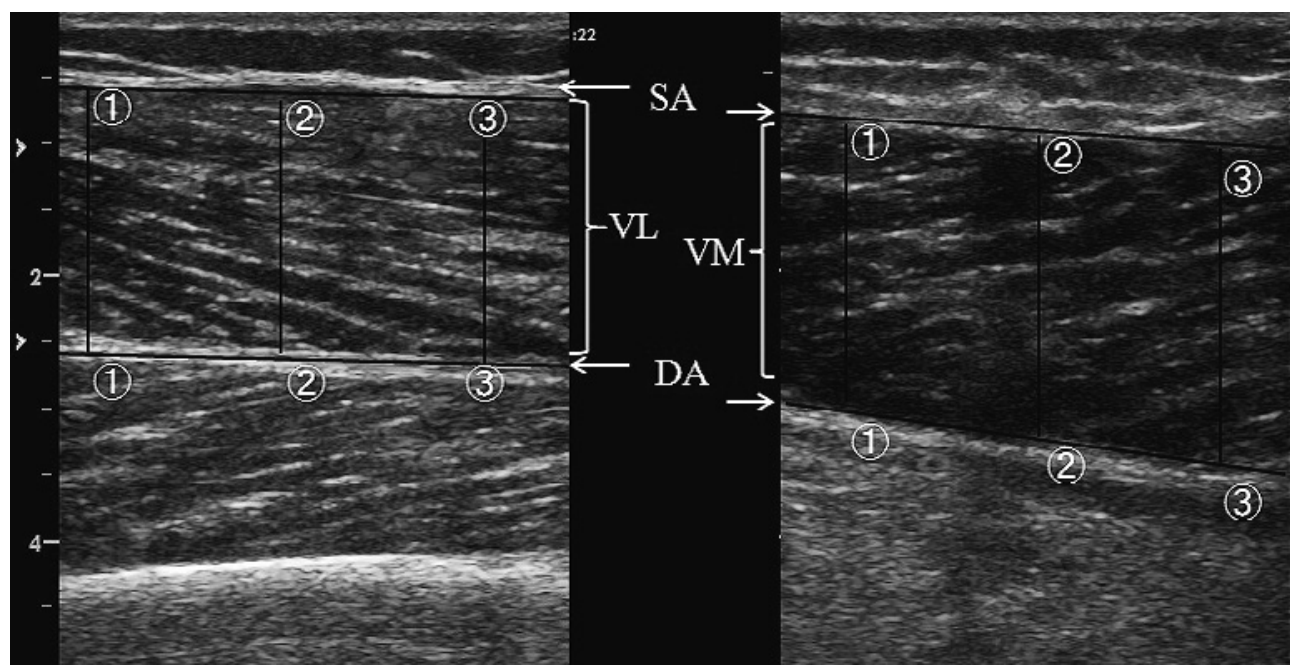

Figure 3.

Measurement of the muscle thickness of the vastus lateraris (VL) and vastus medialis (VM). Muscle thickness was calculated as the length between superficial aponeurose (SA) and deep aponeurose (DA) at three different points (1-1, 2-2, and 3-3) of the ultrasonography image. The mean value of three different points were recorded.

the $50 \mathrm{~mm} 8-\mathrm{MHz}$ linear probe. To obtain the ultrasonography finding, the volunteers lay in the supine position with their knee extension at $0^{\circ}$ and muscle relaxed. Two ultrasonography images were recorded at $2 / 3$ on the line from the anterior superior iliac spine to the lateral side of the patella of VL, and $80 \%$ on the line between the anterior superior iliac spine and the joint space in front of the anterior border of the medial ligament of $\mathrm{VM}^{19)}$. Muscle thickness was calculated as the length between the deep aponeurosis and surface aponeurosis at three difference points of the image. Each ultrasonography image was the mean value recorded (Fig. 3). We measured the reliability of the ultrasonography images in this study, resulting in intra-class correlation coefficients of 0.89 and 0.97 for muscle thicknesses of VL and VM, respectively.

\section{Skeletal muscle mass}

Skeletal muscle mass (SMM) was assessed by the bioelectrical impedance analysis (Inbody 430; Biospace). The skeletal muscle mass index (SMI) was determined by the following formula for whole body skeletal muscle mass and height: (cm)

$\mathrm{SMI}=$ whole body skeletal muscle mass $(\mathrm{kg}) /$ height $^{2}$

SMM and fat mass were assessed at 4 hours later after a meal.

\section{Statistics}

All the analyses were performed using statistical software_SPSS Statistics 21.0 (IBM, SPSS Tokyo, Japan). The decline rates of the sEMG spectral for each contraction were calculated using bivariate linear regression to determine MF linear slope. Statistical significance between men and women in FI and peak torque, muscle thickness was determined using a two-tailed unpaired t-test. All values of $\mathrm{p}<0.05$ were considered significant. Data are shown as mean \pm standard deviations (SD).

\section{Results}

Gender difference in muscle fatigue, peak torque, and muscle thickness, SMI

The results demonstrated that during muscle fatigue assessment, the men had a significantly higher FI $(30.38 \pm$ $6.59 \%)$ than the women $(21.95 \pm 9.19 \%)(p<0.05)$. Knee extension peak torque was normalized by body weight, and the women were shown to have a significantly lower peak torque $(1.43 \pm 0.22 \mathrm{Nm} / \mathrm{kg})$ than the men $(1.99 \pm 0.19 \mathrm{Nm} /$ $\mathrm{kg})(\mathrm{p}<0.05)$ in Table 1 . The calculated exponents of the estimated effect size (ES) and $1-\beta$, and $95 \%$ confidence intervals $(95 \% \mathrm{CI})$ using Gpower 3.1.7 for each variable were FI $(E S=1.1,1-\beta=0.82,95 \% \mathrm{CI}=0.3-16.55)$ and peak torque $(\mathrm{ES}=2.7,1-\beta=0.99,95 \% \mathrm{CI}=0.35-0.77)$. Muscle thickness in VL and VM was significantly greater in the men (VL: $2.05 \pm 0.35 \mathrm{~cm}, \mathrm{VM}: 2.12 \pm 0.45 \mathrm{~cm}$ ) than the women (VL: $1.69 \pm 0.26 \mathrm{~cm}, \mathrm{VM}: 1.64 \pm 0.32 \mathrm{~cm}$ ) (VL: ES $=1.17,1-\beta=0.85,95 \% \mathrm{CI}=0.07-2.0, \mathrm{p}<0.05 ; \mathrm{VM}: \mathrm{ES}=$ $1.23,1-\beta=0.86,95 \% \mathrm{CI}=0.1-2.04, \mathrm{p}<0.05$; respectively) (Table 1). SMI was significantly greater in men $(\mathrm{SMI}=$ $30.98 \pm 3.63)$ than in women $(\mathrm{SMI}=20.34 \pm 0.85, \mathrm{ES}=4.03$, $1-\beta=0.99,95 \% \mathrm{CI}=2.07-5.32, \mathrm{p}<0.01)$.

\section{Gender difference in sEMG spectrum changes}

There was a significant linear trend in the decreased MF slope in VL for the men $(y=-0.3777 x+81.391$, adjusted $\mathrm{R}^{2}=0.30, \mathrm{p}<0.01$ ). The men's VM and women's VL 
Table 1. Male and female characteristics (mean and standard deviations) for FI and peak torque and VL and VM muscle thickness.

\begin{tabular}{lrccc}
\hline & Men $(\mathrm{n}=10)$ & Women $(\mathrm{n}=9)$ & ES & \multicolumn{1}{c}{$95 \%$ CI } \\
\hline FI & $30.38 \pm 6.59 *$ & $21.95 \pm 9.19$ & 1.1 & $0.003-1.92$ \\
Peak torque $(\mathrm{Nm} / \mathrm{kg})$ & $1.99 \pm 0.19 *$ & $1.43 \pm 0.22$ & 2.7 & $0.35-0.77$ \\
VL muscle thickness $(\mathrm{cm})$ & $2.05 \pm 0.35 *$ & $1.69 \pm 0.26$ & 1.17 & $0.07-2.0$ \\
VM muscle thickness $(\mathrm{cm})$ & $2.12 \pm 0.45 *$ & $1.64 \pm 0.32$ & 1.23 & $0.1-2.04$ \\
\hline
\end{tabular}

*Male significantly greater than females $(\mathrm{p}<0.05)$. FI, fatigue index. VL, vastus lateralis. VM, vastus medialis. ES, effect size. $95 \%$ CI, 95\% confidence interval.
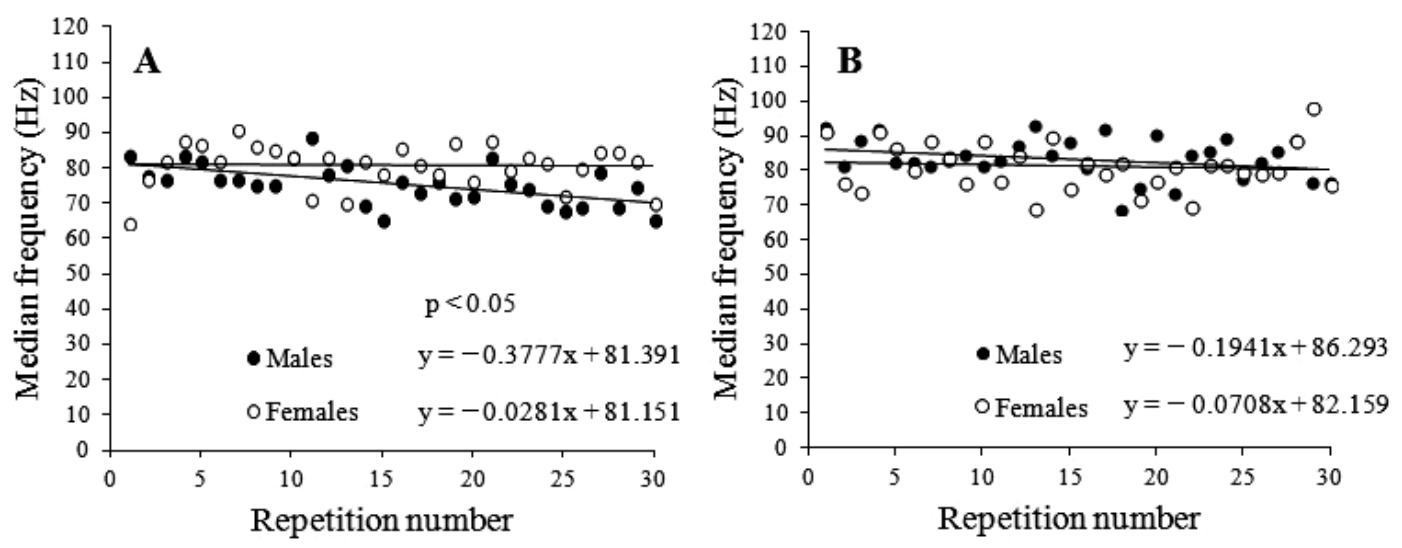

Figure 4.

A: Gender difference of median frequency in the vastus lateralis. There was a significant difference in the decrease in median frequency slope between men and women (slope $=-0.3777$, slope $=-0.0281$, respectively) $(\mathrm{p}<0.05)$. B: There was no significant difference between men and women when assessing the decrease in median frequency slope in the vastus medialis (slope $=-0.1941$, slope $=-0.0708$, respectively) $(\mathrm{p}>0.05)$. Data are shown as mean of median frequency.

and VM trended to have a linear decreased MF slope. However, there were not significant $(\mathrm{y}=-0.1941 \mathrm{x}+86.293$, adjusted $\mathrm{R}^{2}=0.05, \mathrm{p}=0.13 ; \mathrm{y}=-0.0279 \mathrm{x}+81.146$, adjusted $\mathrm{R}^{2}$ $=-0.03, \mathrm{p}=0.83 ; \mathrm{y}=-0.078 \mathrm{x}+82.159$, adjusted $\mathrm{R}^{2}=-0.03, \mathrm{p}$ $=0.63$; respectively). The results demonstrated that for the MF slope in VL during muscle assessment, the men were shown to have a significantly greater decrease in slope than the women (men slope: $-0.31 \pm 0.24$, female slope: -0.003 $\pm 0.37, \mathrm{p}<0.05, \mathrm{ES}=1.04,1-\beta=0.81,95 \% \mathrm{CI}=-0.61-$ $-0.014)$. For the MF slope in VM, gender did not have a significant role (male slope: $-0.112 \pm 0.25$, female slope: $-0.101 \pm 0.21, \mathrm{p}=0.918, \mathrm{ES}=0.05,1-\beta=0.5,95 \% \mathrm{CI}=$ $-0.946-0.855)$ (Fig. 4).

\section{Discussion}

Our results support the hypothesis that men have a significantly greater muscle thickness and knee extension peak torque and a higher decrease in the MF slope than women. Moreover, our findings indicated that muscle fatigue was more affected by a decrease in the MF slope and greater knee extension peak torque and skeletal muscle thickness.

Gender difference in muscle fatigue in the quadriceps femoris muscle has been observed in other studies, and men have a significantly greater percentage decrease of muscle fatigue than women. Pincivero et al. ${ }^{6}$ examined and reported that the knee extensor peak torque and FI in men and women were $2.11 \mathrm{Nm} / \mathrm{kg}$ and $1.53 \mathrm{Nm} / \mathrm{kg}$ for peak torque and $39.35 \%$ and $33.52 \%$ for FI, respectively. Moreover, the gender difference in this present study was similar to those of previous studies that examined muscle fatigue and peak torque $^{6,7)}$. Quadriceps muscle fatigue by the negative slope of knee extensor peak torque, over 30 maximal effort contractions, higher absolute torque values will contribute to a greater per-repetition decrease ${ }^{6}$. Therefore, our results suggest that men have a significantly greater muscle fatigue and peak torque than women.

Our results indicated that male muscle thickness is greater than female muscle thickness for VL and VM. This finding may be explained by the relationship between muscle thickness and muscle strength. In general, maximal muscle strength was reported to correlate with increases in muscle thickness in vivo and in patients ${ }^{20,21)}$. Moreover, cross-sectional muscle is associated with isometric and isokinetic muscle strength ${ }^{22,23)}$.

Recently, sEMG spectral power measurement during muscle fatigue assessment has been reported using wavelet analysis. These studies have shown that MF or mean fre- 
quency spectrum decreases during maximal knee extension effort in the quadriceps. The shift of MF power spectra toward lower frequencies decreases with muscle fatigue. This MF power spectra shift is attributed to muscle lactic acidification $^{24)}$ and potassium accumulation ${ }^{25)}$ with reduced intracellular $\mathrm{pH}$ and decreased muscle fiber conduction velocity $(\mathrm{MFCV})^{26}$, resulting in an accumulation of metabolites ${ }^{27}$. As a result, the decrease in MF and MFCV is thought to be associated with a decrease in fast motor unit firing rates ${ }^{28,29)}$ and recruitment patterns, which results in the synchronization of motor units. MFCV can affect the MF spectrum, such as motor unit firing and synchronization, and recruitment patterns of the motor unit ${ }^{30}$. However, Masuda et al. ${ }^{31}$ reported that MFCV and MF significantly decrease during isometric contraction, but do not decrease during isotonic contractions. In addition, the difference in muscle contraction type has indicated that blood flow and lactic acid accumulation in muscles determines the relationship between $\mathrm{MF}$ and $\mathrm{MFCV}^{26,31)}$. Because dynamic muscle contractions indicate that oxygen supply is maintained through blood flow compared with that in isometric contractions ${ }^{32}$, the decrease of MF during dynamic muscle contractions is not only explained about gender difference by MFCV and intracellular $\mathrm{pH}$ or by accumulation of metabolites. This was because MF slope during muscle fatigue assessment had a similar decrease in men's VL and women's VL. However, this study demonstrated that for the men MF slope was shown to have a significantly greater decrease in slope than women. However, the sEMG signal recorded during muscle contractions was significantly associated with muscle fiber type composition and MF. In addition, Staron et al. ${ }^{33)}$ used muscle biopsies taken from VL of untrained healthy volunteers and reported that muscle fast fiber types are predominant in men, whereas slow fiber types tended to be predominant in women. Interestingly, muscle fiber composition of VL is different from that of VM. Other data have shown that VL and VM of type 2 muscle fiber were $67.3 \%$ and $56.3 \%$, respectively ${ }^{34}$. Moreover, the cross sectional area of type 2 fiber was larger in men compared with women $^{33}$. However, the study design in those studies was specific for the investigation of muscle biopsies for the assessment of gender difference on muscle fiber type. Simoneau JA, et al. ${ }^{35)}$ and Staron RS, et al. ${ }^{33)}$ reported that women VL muscle has been demonstrated that exhibit a greater proportion and smaller type 1 muscle fibers than men, whereas type 1 fibers tended to be the largest in women. Green et al. ${ }^{36)}$ reported that women have a significantly lower overall capacity for aerobic oxidation and anaerobic glycolysis compared with men. Furthermore, glycolytic potential is lower in women. In the present study, the women's MF slope may be explained by the lower glycolytic potential (lower accumulation of metabolites compared with men) and maintenance of oxygen supply by dynamic muscle contractions. Consequently, muscle recruit- ment pattern and muscle mass significantly influenced gender difference. Given the dearth of material that can be used to assess change in muscle recruitment and recruitment muscle fiber type during dynamic knee contractions, these specific parameters have not been clarified. In our present study, the men had a significantly greater decrease of the MF slope in VL than the women, but for VM, there was no significant gender difference in MF. Our results suggest that the cause of muscle fatigue between genders was influenced by the decrease of the MF slope in VL than in VM.

Our results demonstrated that sEMG data were not significant linear trend in the MF slope in women. Women's sEMG spectral data suggest that MFCV was affected by skin thickness, skin temperature, and tissue thickness ${ }^{37)}$. Farina et al. reported that women's muscle is covered by a thicker skinfold than men's muscle ${ }^{38}$. In the current study, we determined sample size using muscle fatigue index data for comparing specific muscle fatigue between men and women. However, Beretta-Piccoli et al. recruited 29 healthy women for assessment of MFCV using sEMG. Their data indicate that more participants are needed to adequately assess sEMG in women. We recommend that studies aiming to assess women sEMG data include a larger number of participants and calculate sample size using women sEMG data.

A potential limitation of the study is the exclusion of elderly volunteers and injured or diseased patients; thereby, the generalization of the study is limited. Furthermore sample size is small for attempting to determine any relationship between muscle fatigue, MF, and muscle thickness. This is because sample size was calculated using FI of nonpaired $t$ test to assess gender difference. Thus, further studies are necessary to clarify the relationship between FI and MF and muscle thickness between males and females. However, our present results indicated that further research using safer and simpler muscle assessment techniques will be of great importance in generalizing the results. Physical and occupational therapists engaged in rehabilitation should be aware of gender difference during resistance training or muscle assessment pertaining to the quadriceps.

A limitation of our study is that recording sEMG signals during dynamic contractions did not take into consideration the movement of the neuromuscular junction of muscle beneath the recording electrodes and changes in muscle length. Further investigation using multi-electrode array and motor unit decomposition technique studies is required to identify the neuromuscular junction of muscle and recording electrodes positions.

\section{Conclusions}

In conclusion, this study provides a gender difference during muscle fatigue assessment where men had a significantly greater muscle thickness and knee extension peak 
torque and a greater decrease of the MF slope in VL than women. In addition, our results indicate that specific muscle fatigue observed during repeated muscle knee contractions associated with gender difference is influenced by the decrease of the MF slope in VL and knee extension peak torque and muscle thickness.

\section{References}

1) Pincivero DM, Campy RM, et al.: Influence of contraction intensity, muscle, and gender on median frequency of the quadriceps femoris. J Appl Physiol (1985). 2001; 90: 804-810.

2) Cioni R, Giannini F, et al.: Sex differences in surface EMG interference pattern power spectrum. J Appl Physiol (1985). 1994; 77: 2163-2168

3) Bishop P, Cureton K, et al:: Sex difference in muscular strength in equally-trained men and women. Ergonomics. 1987; 30: 675687.

4) Maughan RJ, Harmon M, et al.: Endurance capacity of untrained males and females in isometric and dynamic muscular contractions. Eur J Appl Physiol Occup Physiol. 1986; 55: 395-400.

5) Morrow JR Jr and Hosler WW: Strength comparisons in untrained men and trained women athletes. Med Sci Sports Exerc. 1981; 13: 194-197.

6) Pincivero DM, Gandaio CM, et al.: Gender-specific knee extensor torque, flexor torque, and muscle fatigue responses during maximal effort contractions. Eur J Appl Physiol. 2003; 89: 134141.

7) Pincivero DM, Coelho AJ, et al.: Perceived exertion and maximal quadriceps femoris muscle strength during dynamic knee extension exercise in young adult males and females. Eur J Appl Physiol. 2003; 89: 150-156.

8) Henriksson-Larsén K: Distribution, number and size of different types of fibres in whole cross-sections of female $\mathrm{m}$ tibialis anterior. An enzyme histochemical study. Acta Physiol Scand. 1985; 123: 229-235.

9) Simoneau JA and Bouchard C: Human variation in skeletal muscle fiber-type proportion and enzyme activities. Am J Physiol. 1989; 257: E567-572.

10) von Tscharner V: Intensity analysis in time-frequency space of surface myoelectric signals by wavelets of specified resolution. J Electromyogr Kinesiol. 2000; 10: 433-445.

11) Pincivero DM, Gear WS, et al.: Assessment of the reliability of high-intensity quadriceps femoris muscle fatigue. Med Sci Sports Exerc. 2001; 33: 334-338.

12) Karlsson S, Yu J, et al.: Time-frequency analysis of myoelectric signals during dynamic contractions: a comparative study. IEEE Trans Biomed Eng. 2000; 47: 228-238.

13) So RC, Ng JK, et al: : EMG wavelet analysis of quadriceps muscle during repeated knee extension movement. Med Sci Sports Exerc. 2009; 41: 788-796.

14) Beck TW, Stock MS, et al.: Shifts in EMG spectral power during fatiguing dynamic contractions. Muscle Nerve. 2014; 50: 95102.

15) Stock MS, Beck TW, et al.: Sex comparisons for relative peak torque and electromyographic mean frequency during fatigue. Res Q Exerc Sport. 2013; 84: 345-352.

16) Kupa EJ, Roy SH, et al.: Effects of muscle fiber type and size on EMG median frequency and conduction velocity. J Appl Physiol. 1985; 79: 23-32.

17) Sadoyama T, Masuda T, et al.: Fibre conduction velocity and fiber composition in human vastus lateralis. Eur J Appl Physiol Occup Physiol. 1988; 57: 767-771.

18) Lindström B, Karlsson S, et al.: Knee extensor performance of dominant and non-dominant limb throughout repeated isokinetic contractions, with special reference to peak torque and mean frequency of the EMG. Clin Physiol. 1995; 15: 275-286.

19) Merletti R and Hermens $H$ : Introduction to the special issue on the SENIAN European concerted action. J Electromyogr Kinesiol. 2000; 10: 283-286.

20) Freilich RJ, Kirsner RL, et al.: Isometric strength and thickness relationships in human quadriceps muscle. Neuromuscul Disord. 1995; 5: 415-422.

21) Malas FÜ, Ozçakar L, et al.: Effects of different strength training on muscle architecture: clinical and ultrasonographic evaluation in knee osteoarthritis. PM R. 2013; 5: 655-662.

22) Akagi R, Takai Y, et al.: Relationships between muscle strength and indices of muscle cross-sectional area determined during maximal voluntary contraction in middle-aged and elderly individuals. J Strength Cond Res. 2009; 23: 1258-1262.

23) Gibbons LE, Latikka P, et al.: The association of trunk muscle cross-sectional area and magnetic resonance image parameters with isokinetic and psychophysical lifting strength and static back muscle endurance in men. J Spinal Disord. 1997; 10: 398403.

24) Allen DG, Lamb GD, et al.: Skeletal muscle fatigue: cellular mechanisms. Physiol Rev. 2008; 88: 287-332.

25) Fortune E and lowery MM: Effect of extracellular potassium accumulation on muscle fiber conduction velocity: a simulation study. Ann Biomed Eng. 2009; 37: 2105-2117.

26) Zwarts MJ and Stegeman DF: Multichannel surface EMG: basic aspects and clinical utility. Muscle Nerve. 2003; 28: 1-17.

27) Vollestad NK and Sejersted OM: Biochemical correlates of fatigue. Eur J Appl Physiol. 1988; 57: 336-347.

28) Moritani T, Muro M, et al.: Intramuscular and surface electromyogram changes during muscle fatigue. J Appl Physiol. 1986; 60: 1179-1185.

29) Miller RG, Kent-Braun JA, et al.: Mechanisms of human muscle fatigue. Quantitating the contribution of metabolic factors and activation impairment. Adv Exp Med Biol. 1995; 384: 195-210.

30) Lowery MM, Nolan PJ, et al.: Electromyogram median frequency, spectral compression and muscle fiber conduction velocity during sustained sub-maximal contraction of the brachioradialis muscle. J Electromyogr Kinesiol. 2002; 12: 111-118.

31) Masuda K, Masuda T, et al.: Change in surface EMG parameters during static and dynamic fatiguing contractions. J Electromyogr Kinesiol. 1999; 9: 39-46.

32) Hultman E and Sahlin K: Acid-base balance during exercise. In: Wilmore JH and Keogh JF (eds): Exercise and sport sciences reviews, vol. 8. Academic Press, New York, 1980, pp. 41-128. 
33) Staron RS, Hagerman FC, et al.: Fiber type composition of the vastus lateralis muscle of young men and women. J Histochem Cytochem. 2000; 48: 623-629.

34) Johnson MA, Polgar J, et al.: Data on the distribution of fibre types in thirty-six human muscles. J Neurol Sci. 1973; 18: 111129.

35) Simoneau JA and Bouchard C: Human variation in skeletal muscle fiber-type propotion and enzyme activities. Am J Physiol. 1989; 257: 567-572.

36) Green HJ, Fraser IG, et al.: Male and female differences in en- zyme activities of energy metabolism in vastus lateralis muscle. J Neurol Sci. 1984; 65: 323-331.

37) Bilodeau M, Schindler-Ivens S, et al.: EMG frequency content changes with increasing force and during fatigue in the quadriceps femoris muscle of men and women. J Electromyogr Kinesiol. 2003; 13: 83-92.

38) Farina D, Macaluso A, et al.: Effect of power, pedal rate, and force on average muscle fiber conduction velocity during cycling. J Appl Physiol. 2004; 97: 2035-2041. 